\title{
Epithelial branching: the power of self-loathing
}

\author{
Wen-Chin Lee ${ }^{1}$ and Jamie A. Davies ${ }^{2 *}$
}

\begin{abstract}
Summary
Branching morphogenesis of epithelia is an important mechanism in mammalian development. The last decade has seen the identification of many signalling pathways and intracellular mechanisms that control epithelial branching. Tissue-level mechanisms that space new branches out have, however, remained an unsolved problem. A recent publication by Nelson et al. ${ }^{(1)}$ suggests-if extrapolation from their novel and abstract culture system is valid-that branches may be spaced out by a system of mutual inhibition based on diffusion of TGF $\beta$. Such a system would allow a developing tree to arrange itself, without detailed genetic specification, by adaptive self-organization. BioEssays 29:205-207, 2007. (c) 2007 Wiley Periodicals, Inc.
\end{abstract}

\section{Introduction}

Many aspects of animal physiology depend on the existence of large areas of surface for exchange of substances between the tissues and the outside of the body. A common solution to the problem of maximising this surface area is for an organ to be tightly packed with units specialized for substance exchange (air alveoli, milk alveoli, urinary nephrons etc), and for these to be connected to the outside world by a tree-like system of branched epithelial tubes (tracheae, milk ducts, urinary collecting ducts etc). The formation of these epithelial trees, by branching morphogenesis, is therefore a ubiquitous mechanism of mammalian development. ${ }^{(2)}$

An important unsolved problem in branching morphogenesis is undertsanding the mechanism by which the branches of the tree space themselves out appropriately. Evidence from the variable micro-anatomies of each organ, from the anatomical flexibilty of the developing epithelia in different organ culture systems and also probably from common sense argues against the existence of a rigid genetic program that specifies the final length and angle of divergence of every branch of every generation of branching exactly. Rather, it has been argued, by us and by others, ${ }^{(3,4)}$ that the genetic

\footnotetext{
${ }^{1}$ Chang Gung Memorial Hospital-Kaohsiung Medical Center, Taiwan.

${ }^{2}$ University of Edinburgh, UK.

${ }^{*}$ Correspondence to: Jamie A. Davies, Centre for Integrative Physiology, University of Edinburgh, Hugh Robson Building, George Square, Edinburgh, Scotland EH8 9XB, UK.

E-mail: jamie.davies@ed.ac.uk

DOI 10.1002/bies.20541

Published online in Wiley InterScience (www.interscience.wiley.com).
}

programme specifies a cellular machinery that enables the growing tree to make its own appropriate decisions about where and when to initate new branches.

In all examples of epithelial branching so far studied, morphogenesis is dependent on ramogenic (branchpromoting) growth factors released from surrounding tissues. Speculation about the spacing mechanisms has therefore tended to concentrate on these paracrine signals, a line of thought that has received some encouragement from genetic evidence in Drosophila melanogaster. Each body segment of $D$. melanogaster is equipped with a system of branched airtubes that conduct air from the outside of the body to the internal muscles and other tissues that require it. The branching of these tracheae depends on the diffusible FGF homologue Branchless, produced by other tissues, that acts through the FGFR homologue Breathless on the epithelia (these molecules are named after the phenotypic effects of their absence). The efficiency with which this signal is transduced inside the cell is modulated by the activity of another protein, Sprouty. Absence of functional Sprouty causes a dramatic increase in branching, so that what should be a controlled tree becomes an uncontrolled spiky mess; this is exactly what would be expected if the normal spacing mechanisms failed. Sprouty proteins are expressed in mammalian organs too, but their disruption fails to cause such a dramatic phenotype and those hoping that this fruitfly story would lead directly to the spacing mechanisms in mammals have been disappointed.

It is against this background that Nelson et al. ${ }^{(1)}$ have used a novel cell culture system to investigate branch-spacing mechanisms in the mammary gland, and have suggested that epithelium-epithelium inhibitory signals, mediated by TGF $\beta$, may be the critical regulators of the shape of the developing tree.

\section{The culture system of Nelson et al.}

The culture system used by Nelson et al. builds on a method of studying epithelial branching morphogenesis that was pioneered in the early 1990s by Santos and Nigam. ${ }^{(5)}$ In this system, epithelial cells (renal, in that seminal paper) are suspended in collagenous three-dimensional matrices and treated with growth factors that encourage them to produce cysts and then tubules that elongate and branch. To this basic system, Nelson et al. added a series of elastomeric stamps that can be used, rather like a gel comb, to produce wells of 
defined shape, size and spacing in the gel. They then placed mouse mammary epithelial $(\mathrm{EpH} 4)$ cells, primary mammary cells or primary mammary organoids (cyst-like aggregates of polarized cells and myoepithelia) in these wells and sealed the tops with a second layer of collagen gel. The cells formed hollow tubes that lined the edges of their wells. After incubation, the arrangement of the cells, including any invasion of the gel, was visualized by confocal microscopy. The highly controlled geometry of this system allowed the authors to investigate the effect of space on development.

\section{Invasion of the gel points away from existing cells}

With this novel culture system, classical staining techniques, frequency maps and three-dimensional models of diffusion, Nelson et al. first characterized the behaviour of mammary cells, allowed to form hollow tubes in rectangular wells and then treated with ramogens such as epidermal growth factor (EGF) or hepatocyte growth factor (HGF). They found that cells invaded the gel (apparently as mesenchyme-like, vimentin-positive migratory cells rather than as epithelia), but that invasion took place only from the ends, rather than the sides, of the wells (Fig. 1). Epithelial (vimentin-) to mesenchymal (vimentin+) transition (EMT) preceded overt invasion.

To explore further the reason for EMT and invasion being restricted to the ends of rectangular wells, the authors experimented with different-shaped wells, such as $C$ and $Y$ shapes. In the $Y, E M T$ and invasion was again from the ends of the tubules and, in the $\mathrm{C}$ shapes, it was from the left, but not the right, edge of the ' $C$ ' as it appears on this page. In other words, it was always in the direction that took the cells away from other cells. Combinations of nearby wells also respected the rule of invasive EMT being suppressed if it would be taking place close to other cells. This suggested the action of an autocrine inhibitor. Using computer-modeling techniques and different geometries, Nelson et al. found that the site of EMT and invasion was determined by tubule geometry and was consistent with the predicted concentration profiles of secreted diffusible inhibitors.

\section{TGF $\beta$ as a controller of patterning}

TGF $\beta$ has been known for many years to be an inhibitor of epithelial branching, this role having been demonstrated in the original collagen matrix paper of Santos and Nigam ${ }^{(5)}$ and also in kidneys, lungs and mammary glands (reviewed in a previous issue of this journal $\left.{ }^{(6)}\right)$. Nelson et al. therefore considered it as a candidate for the inhibitor of invasive EMT in their system. They confirmed the expression of TGF $\beta$ family ligands and receptors by RT-PCR. Significantly, the authors managed to demonstrate, by immunofluorescence staining, a concentration gradient of TGF $\beta 1$ emanating from the cells that was compatible with the numerical predictions of their computer model. All attempts to disrupt TGF $\beta$ signalling, including TGF $\beta 1$ antibody, pharmacological inhibition of TGF $\beta$ type I receptor kinase and overexpression of a dominant negative TGF $\beta$ type II receptor, successfully blocked the inhibition and resulted in invasive EMT from all surfaces of the wells.

Together, these data suggest a model in which cells respond positively to exogenous ramogens and negatively to the TGF $\beta$ that they themselves make. The outcome is that epithelial cells are most likely to undergo invasive EMT where the concentration of TGF $\beta$ is lowest, which is where the proximity of other epithelial cells is also lowest. In this way, invasion will naturally be directed to uninvaded space, and the cell population will spread as a ramifying tree rather than a spherical balloon; the 'automatic arrangement' mechanism that results from this would be an excellent example of adaptive self-organization. ${ }^{(3)}$

\section{From gels to organs?}

At first glance, this excellent paper seems to have answered the old question posed at the beginning of this article, and provided the mechanism responsible for the spacing of an epithelial tree. Its interpretation as a model for
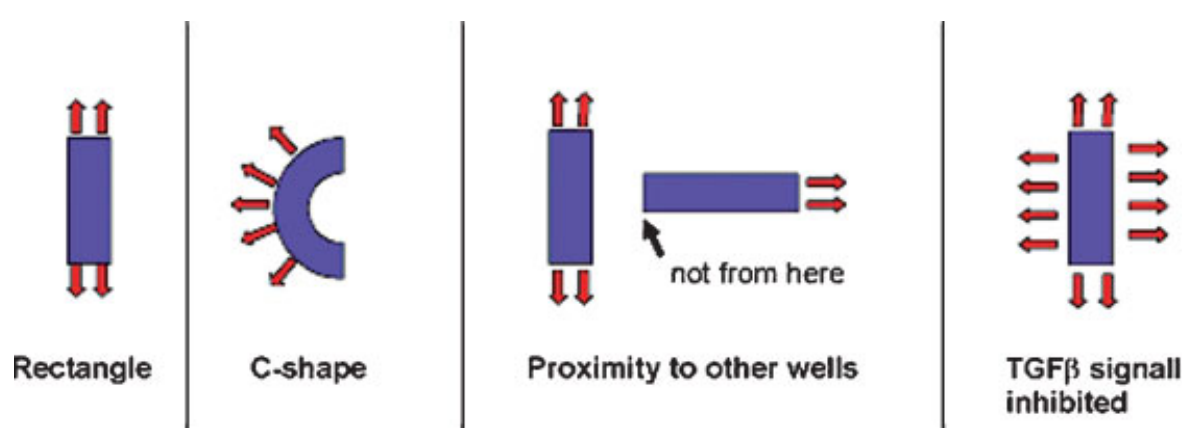

TGF $\beta$ signalling inhibited

Figure 1. Invasive EMT (red arrows) from epithelial tubules (blue blocks) growing in different controlled geometries. Except when signalling by TGF $\beta$ in inhibited, invasion is directed towards zones of the gel in which diffusible inhibitors released by the epithelium are least concentrated. 
branching morphogenesis does, however, rely on a number of assumptions.

One assumption is that the epithelium-in-gel culture system is a valid abstraction of the normal mammary gland, in which the epithelium is surrounded by mesenchymal cells. The mesenchyme is known to exchage numerous signals with the epithelium and may also contribute mechanically to the development of the tissue: it is possible that, in the complete organ, the mesenchymal influences dominate pattern. Nevertheless, the fact that epithelium can organize itself in a simple gel culture system is itself an argument that this ability may be involved somehow in the complete organ. It may also be relevant to mention that, working with micromanupilated kidney rudiments, we have obtained preliminary evidence for mutual repulsion of branching epithelia even with mesenchyme present (Sweeney, Lindstrom, Davies unpublished).

Another critical assumption is that invasive EMT is a valid proxy for epithelial branching. A previous review by the laboratory responsible for the Nelson et al. paper speculated that transient EMT at the tips of the epithelial tree may assist the invasion of these tips through the fat pad. ${ }^{(7)}$ There is, however, as yet no real evidence that this happens, and a recent (2006) review has stated that "there is no real evidence that a transient EMT takes place during mammary branching...". (4) Indeed, EMT from mammary organoid cultures is used by other researchers as a proxy for malignant invasion (as in mammary carcinoma) rather than normal development. ${ }^{(8-10)}$ It is possible, therefore, that Nelson et al. have actually uncovered a mechanism that results in efficient invasion of tissue by metastatic cells rather than-or as well as - the mechanism that spaces out an epithelial tree. Sorting out the connection, if any, between branching in whole organs and EMT in collagen gels will depend on better knowledge of the intracellular pathways that control both of them.

These caveats and further questions should not detract from a study that is both clever and original. The combination of a novel, geometrically controlled culture system, numerical simulation and direct detection of protein gradients in situ makes a powerful system, and this paper is an important contribution to an important and growing field.

\section{References}

1. Nelson CM, Vanduijn MM, Inman JL, Fletcher DA, Bissell MJ. 2006 Tissue geometry determines sites of mammary branching morphogenesis in organotypic cultures. Science 314:298-300.

2. Davies J. 2005. Branching morphogenesis. Landes Biomedical.

3. Davies J. 2005. Mechanisms of Morphogenesis, Elsevier Academic Press, London

4. Lu P, Sternlicht MD, Werb Z. 2006. Comparative Mechanisms of Branching Morphogenesis in Diverse Systems. J Mammary Gland Biol Neoplasia 11:213-228.

5. Santos OF, Nigam SK. 1993. HGF-induced tubulogenesis and branching of epithelial cells is modulated by extracellular matrix and TGF-beta. Dev Biol 160:293-302.

6. Davies JA. 2002. Do different branching epithelia use a conserved developmental mechanism? Bioessays 24:937-948.

7. Fata JE, Werb Z, Bissell MJ. 2004. Regulation of mammary gland branching morphogenesis by the extracellular matrix and its remodeling enzymes. Breast Cancer Res 6:1-11.

8. McLachlan E, Shao Q. Wang HL, Langlois S, Laird DW. 2006. Connexins act as tumor suppressors in three-dimensional mammary cell organoids by regulating differentiation and angiogenesis. Cancer Res 66:98869894

9. Nouhi Z, Chughtai N, Hartley S, Cocolakis E, Lebrun JJ, Ali S. 2006 Defining the role of prolactin as an invasion suppressor hormone in breast cancer cells. Cancer Res 66:1824-1832.

10. Robson EJ, Khaled WT, Abell K, Watson CJ. 2006. Epithelial-tomesenchymal transition confers resistance to apoptosis in three murine mammary epithelial cell lines. Differentiation 74:254-264. 